\title{
A Study on the L2 Stability and Transparency of Three Channel Control Architectures in Bilateral Teleoperation under Time Delays
}

\author{
Zaman Gecikmeleri Altında İki-Yönlü Teleoperasyonda Üç Kanallı Kontrol \\ Mimarilerinin L2 Kararlılığı ve Şeffaflı̆̆ Üzerine Bir Çalışma
}

\author{
Uğur TÜMERDEM ${ }^{1}$ \\ ${ }^{1}$ Mechanical Engineering Department Marmara University, 34722, Istanbul, Turkey
}

\begin{abstract}
Bilateral/haptic teleoperation is the remote control of a "slave" robotic system through a "master" robot or haptic interface, and involves the feedback of the slave interaction forces to the operator. Thus, the master operator can operate the slave as an extension of his/her body. Time delay among the robots is a long-standing problem in bilateral teleoperation. The existence of force feedback to the operator in haptic/bilateral teleoperation makes the teleoperation system less robust to time delays on the communication channels. Three channel architectures have been proposed in the literature to provide increased robustness against time delays with increased transparency (kinesthetic coupling). In this paper, we propose modifications on three channel architectures to guarantee delay independent L2 stability, while exploiting the increased transparency characteristics of these architectures. The validity of the proposed approach is examined both analytically and experimentally on a bilateral teleoperation system.
\end{abstract}

Keywords: Robotics, Teleoperation, Haptics, Force Feedback, Stability

Öz

İki-yönlü/haptik teleoperasyon; bir "takipçi" robotun bir "yönlendirici" robot, veyahut arayüz, aracıllı̆ıyla operatör tarafindan uzaktan kumandasını ve takipçi robotun etkileşim kuvvetlerinin operatöre geri beslenmesini kapsar. Böylece operatör, takipçi robotu kendi uzuvlarının bir uzantısı gibi kullanabilir. Robotlar arasındaki iletişimde yaşanan gecikmeler, iki-yönlü teleoperasyonda bilinen en eski problemlerdendir. Operatöre kuvvet geri beslemesinin olması haptik/iki-yönlü teleoperasyon sistemlerinin gecikmelere karşı daha kararsız/dayanıksız olmasına sebebiyet vermektedir. Üç kanal mimarileri, literatürde, gecikmelere karşı daha dayanıklı ve yüksek transparanlığa (kinestetik bağa) izin veren mimariler olarak ortaya çıkmışlardır. $\mathrm{Bu}$ makalede, üç kanal mimarilerinde, bu mimarilerin hem transparanlığından faydalanmayı amaçlayan hem de gecikmeden bağımsız L2 kararlılığını garantileyen değişiklikler yapılması önerilmektedir. Önerilen yaklaşımın geçerliliği hem analitik hem de deneysel yöntemlerle bir iki-yönlü teleoperasyon sisteminde incelenmiş, ve doğrulanmıştır.

Keywords: Robotik, Teleoperasyon, Haptik, Kuvvet kontrolü, Kararlılık

\section{INTRODUCTION}

Teleoperation systems were first developed for remote manipulation in nuclear plants, by Goertz. The first teleoperation systems were composed of master and slave manipulators that were mechanically coupled. Later Goertz also led the development of first electrically controlled teleoperator systems where the master and slave manipulators were connected to each other through electrical communication. This development has also expanded the application fields of teleoperator systems, to military and law enforcement, aerospace, medical and construction. Bilateral teleoperation systems involve actuated master systems, in contrast with unilateral teleoperation systems with passive masters, which can feed the forces acting on the slave systems back to the operator first by acquiring the force measurement with a sensor and then transmitting this measurement back.

One of the greatest problems in bilateral teleoperation systems is the existence of time delays as the presence of force feedback coupled with time delay destabilizes the control system. The first solution to the time delay problem in bilateral teleoperation was presented in [1] by Anderson and Spong, by treating the communication among the master and the slave as a power transmission line and rendering the overall system passive by using the scattering approach. The passivity concept was further developed with the use of wave variables in [2] by Niemeyer and Slotine. Many other researchers [3][4][5] have since used and built upon the passivity approach to guarantee stability of bilateral teleoperation systems under time delay. However, an important drawback of the passivity approach is that the performance of the control system deteriorates once it is made passive. The performance of bilateral teleoperation systems is often described by the term "transparency". Transparency refers to the teleoperation system being transparent to the operator, thus enabling perfect kinesthetic coupling between the 
operator and the environment. One way to quantify transparency is to use hybrid matrices as proposed by Hannaford [6]. Lawrence [7] and Yokokohji [8] have independently shown that perfect transparency can be achieved by using the four channel control architecture, that makes use of four communication channels among the master and slave robots: two position channels for the position control loops of each robot and two force channels for the force control of each robot. However it is widely known that the four channel architecture is not capable of guaranteeing stability and transparency under time delays. It was later shown that the transmission of four measurements among the robots as in the four channel architecture is not a requisite for transparency. By using local force compensators, one of the force communication channels can be eliminated, and transparency can still be achieved when there is no time delay with a three channel architecture [9][10]. Furthermore, three channel architecture offers better transparency than the four channel architecture under time delay. In [11], Kubo et. al. has proposed a novel three channel approach eliminating one of the position channels rather than the force channels, to provide some improvements in terms of transparency under time delay. In [12], a type of three channel architecture has been applied to multilateral teleoperation with multiple robots. In [13], passivity/ absolute stability and Z-width (transmitted impedance bandwidth) based transparency analyses of all three channel architectures presented in [9]-[11] have been performed. However, it has been shown that it is impossible to guarantee absolute stability of any of the three channel architectures for all frequencies. Furthermore, Z-width does not provide a complete picture of the critical kinematic/force coupling performance of the teleoperation system.

The reason that three channel architectures can not guarantee absolute stability is that, absolute stability/passivity criteria are often too conservative. In this paper we are proposing the use of input-output L2 stability [14][15] for the stability analysis of three channel teleoperation systems due to more relaxed stability conditions. It will be shown that by introducing modifications on the known three channel architectures it is possible to guarantee delay independent stability of all the three channel controllers for all LTI environmental parameters for the first time. Furthermore we also analyze the effects of the proposed stability modifications on the transparency of the system under time delay. For transparency analyses/comparisons of all three channel architectures, hybrid and inverse hybrid matrices are utilized, which also allow the quantification of kinematic/force correspondences. The theoretical results are then validated with experiments performed on a bilateral teleoperation system composed of two linear motors.

The organization of the paper is as follows: Section II describes the Lawrence and three channel architectures for bilateral teleoperation under time delay and introduces the proposed modifications, Section III provides the stability analysis and Section IV provides transparency analyses for the proposed framework, Section V reports the experimental results and Section VI discusses the outcomes of this research and possible future directions.

\section{THREE CHANNEL ARCHITECTURES}

In bilateral teleoperation, Lawrence architectures [16] are commonly used to obtain controllers. This name stems from the four channel architecture proposed by Lawrence and by using the channel parameters described in that paper almost all teleoperation controllers can be obtained.. While describing the three channel architecture Zaad and Salcudean [9] proposed the use of two local force compensation channels. Here we will be using the same approach to describe the dynamic equations of a bilateral teleoperation system. The dynamic equations of the master and the slave systems under the mentioned control laws are given respectively in frequency domain:

$$
\begin{aligned}
& \left(Z_{m}+C_{m}(s)\right) V_{m}(s)-C_{4} V_{s}(s) e^{\left\{-s T_{2}\right\}} \\
& =\left(1-C_{6}\right) F_{m}(s)-C_{2} F_{s}(s) e^{\left\{-s T_{2}\right\}} \\
& \left(Z_{s}+C_{s}(s)\right) V_{s}(s)-C_{1} V_{m}(s) e^{\left\{-s T_{1}\right\}} \\
& =\left(C_{5}-1\right) F_{s}(s)+C_{3} F_{m}(s) e^{\left\{-s T_{1}\right\}}
\end{aligned}
$$

Here, $\mathrm{s}$ is the complex frequency variable, $V_{m}, V_{s}$ are the velocity vectors for the master and slave robots, $Z_{m}, Z_{s}$ are the impedances of each robot. The impedances are assumed to be masses $Z_{m}=M_{m} s, Z_{s}=M_{s} s$. $F_{m}(s), F_{s}(s)$ are the forces acting on the master and slave robots respectively. In this paper these forces are modeled as linear time invariant (LTI) systems as in [9]:

$F_{m}=F_{h}^{*}-Z_{h} v_{m}$
$F_{s}=F_{e}^{*}+Z_{e} v_{s}$

$Z_{h}, Z_{e}$, are the human and environmental impedance terms respectively and can be written as: $Z_{h}=m_{h} s+$ $b_{h}+\frac{k_{h}}{s}, Z_{e}=m_{e} s+b_{e}+\frac{k_{e}}{s}$ where $m_{h, e}, b_{h, e}, k_{h, e}$ are the human and environmental mass, spring and damper coefficients respectively. $F_{h}^{*}, F_{e}^{*}$ are the exogenous master and environmental forces which are independent of the system states. $C_{m}(s), C_{s}(s)$ are the local position feedback controllers, $C_{1}(s), C_{4}(s)$ are position channel feedforward controllers, $C_{2}, C_{3}$ are force channel feedforward control gains, and $e^{\left\{-s T_{1}\right\}}, e^{\left\{-s T_{2}\right\}}$ are the time delays between each robot where $T_{1}$ is the amount of time delay in seconds from the master robot to the slave and $T_{2}$ is the delay from the slave to the master. Here the robots are assumed to behave as pure masses in the Cartesian space, and disturbance observers and or computed torque method can be utilized to transform any robot dynamics into 
this form. The control diagram can then be seen in Figure 1.

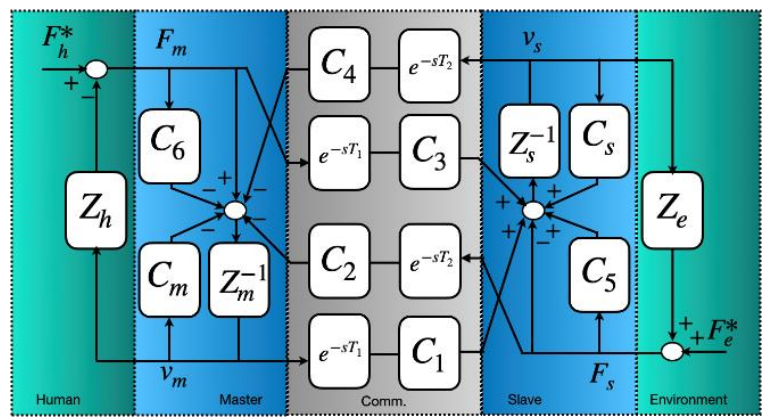

Figure 1. Lawrence architectures in Bilateral Teleoperation

Conventional four channel controllers are obtained by the following selection of the controller/channel parameters:

$$
\begin{aligned}
& C_{1}(s)=C_{4}(s)=C_{m}(s)=C_{s}(s)=k_{p} / s+k_{v} \\
& C_{2}=C_{3}=1 \\
& C_{5}=C_{6}=0
\end{aligned}
$$

Three channel architectures as devised by Zaad and Salcudean [9]-[10] make use of the local force feedback channels $C_{5}, C_{6}$ to compensate for the effects of forces acting on one of the robots so that the robot is purely position controlled. This robot can be selected as the master or the slave, depending on the requirements for the teleoperation system (such as environment the slave robot will be in contact with.) This allows the elimination of the force channel to the force compensated/position controlled robot, reducing in effect the number of communication channels to three. In this paper we will call the three channel architecture where the master is position controlled P-PF architecture, and the one where the slave is position controlled the PF-P architecture. This naming convention implies that the PF controlled robot receives both force and position measurements from the other robot and $\mathrm{P}$ controlled robot receives only position information. The channel and controller parameters for the P-PF architecture can be written as follows:

$$
\begin{aligned}
& C_{1}(s)=C_{4}(s)=C_{m}(s)=C_{s}(s)=k_{p} / s+k_{v} \\
& C_{2}=0, C_{3}=1 \\
& C_{5}=0, C_{6}=1
\end{aligned}
$$

On the other hand the PF-P architecture can be written as:

$$
\begin{aligned}
& C_{1}(s)=C_{4}(s)=C_{m}(s)=C_{s}(s)=k_{p} / s+k_{v} \\
& C_{2}=1, C_{3}=0 \\
& C_{5}=1, C_{6}=0
\end{aligned}
$$

Both of these controllers are designed with the assumption that one of the robots is position controlled and the disturbances acting on the robot in the form of external forces are compensated through the control system. However Kubo et al. [11] has also shown that if one of the robots is purely force controlled instead of being position controlled a new kind of three channel architecture can be obtained for transparency. This would mean that both robots communicate their force measurements to one another and one robot also transmits its position measurements. In this architecture the local force compensation channels are also not mandatory. Thus, two more three channel architectures are obtained: the F-PF and PF-F types. The F-PF type three channel architecture can be obtained by the following selection of controllers:

$$
\begin{aligned}
& C_{1}(s)=C_{s}(s)=k_{p} / s+k_{v}, C_{4}(s)=C_{m}(s)=0 \\
& C_{2}=1, C_{3}=1 \\
& C_{5}=0, C_{6}=0
\end{aligned}
$$

The final type of three channel architecture is the PF-F type described by the following channel parameters:

$$
\begin{aligned}
& C_{1}(s)=C_{s}(s)=0, C_{4}(s)=C_{m}(s)=k_{p} / s+k_{v} \\
& C_{2}=1, C_{3}=1 \\
& C_{5}=0, C_{6}=0
\end{aligned}
$$

Linear stability analysis under time delays using Nyquist stability criterion has been demonstrated for the PF-P and P-FP three channel architectures in [10]. Absolute stability analysis has been performed for the F-PF and PP-F architectures in [12], however it has been demonstrated that stability can not be guaranteed for all frequencies. In this paper we will perform delay independent $L_{2}$ stability analysis as proposed in [15] to demonstrate that the architectures with the controllers given as in (8)-(19) can not guarantee delay independent stability. However we will also demonstrate for the first time that with some modifications on the channel and local controllers, it is possible to guarantee delay independent stability for all three channel architectures. We modify the local controllers $C_{m}, C_{s}$ by adding local damping injection terms and introduce force controllers $C_{f}$ which will be selected as proportional controllers for the P-PF,PF-P architectures and as high-pass filters for the F-PF,PF-F architectures. The new local controller parameters $C^{\prime}{ }_{m}, C^{\prime}{ }_{s}$ for the PF-P architecture can then be written as:

$$
C^{\prime}{ }_{s}(s)=C_{s}(s)+b_{s}, C_{m}^{\prime}(s)=C_{m}(s)+b_{m}
$$

and the new force controllers can be written as :

$$
\begin{aligned}
& C_{2}=C_{f}, C_{3}=0 \\
& C_{5}=1, C_{6}=1-C_{f}
\end{aligned}
$$


Here, $b_{m}, b_{s}$ are the local master and slave velocity feedback coefficients employed for damping injection. For the P-PF architecture, the modified controllers can be written as:

$C^{\prime}{ }_{s}(s)=C_{s}(s)+b_{s}, C^{\prime}{ }_{m}(s)=C_{m}(s)+b_{m}$

$C_{2}=0, C_{3}=C_{f}$

$C_{5}=1-C_{f}, C_{6}=1$

For the PF-F and F-PF architectures, force controller will be modified as a high pass filter: $C_{f}{ }^{\prime}(s)=\frac{C_{f} s}{s+g}$. The PF-F architecture controller parameters can then be written as:

$$
\begin{aligned}
& C^{\prime}{ }_{s}(s)=b_{s}, C^{\prime}{ }_{m}(s)=C_{m}(s)+b_{m} \\
& C_{2}=C_{f}{ }^{\prime}, C_{3}=C_{f}{ }^{\prime} \\
& C_{5}=1-C_{f}{ }^{\prime}, C_{6}=1-C_{f}{ }^{\prime}
\end{aligned}
$$

Finally, the F-PF architecture will be written as:

$$
\begin{aligned}
& C^{\prime}{ }_{s}(s)=C_{s}(s)+b_{s}, C^{\prime}{ }_{m}(s)=b_{m} \\
& C_{2}=C_{f}{ }^{\prime}, C_{3}=C_{f}{ }^{\prime} \\
& C_{5}=1-C_{f}{ }^{\prime}, C_{6}=1-C_{f}{ }^{\prime}
\end{aligned}
$$

The reasons for these selections of controllers will be evident in the stability and transparency analyses in the next two sections. Firstly we derive the necessary conditions for delay independent $L_{2}$ stability with these controllers.

\section{INPUT-OUTPUT STABILITY ANALYSIS}

To guarantee stability of the teleoperation system, input-output stability analysis will be performed [17]. An input-output connection refers to two systems interconnected in feedback configuration as in Figure 2. We first start with the definitions and properties of the signal spaces used in this paper. $L_{2}$ space is the set of square integrable functions $f(t)$ defined in the domain $t:[0, \infty)$, satisfying the inequality: $\int_{0}^{\infty}|f(t)|^{2} d t<\infty$. The extended $L_{2}$ spaces $L_{2 e}$ are the set of functions in $L_{2}$ that are equal to 0 for $t>T$, satisfying the inequality: $\int_{0}^{T}|f(t)|^{2} d t<\infty$. The norm of a signal in $L_{2}$ space is given as ||$f||=$ $\left(\int_{0}^{\infty}|f(t)|^{2} d t\right)^{1 / 2}$.

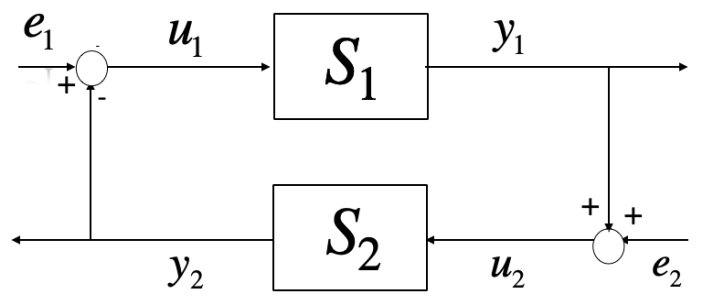

Figure 2. Input-Output connected systems
The equations describing an input-output connected system as in Figure 2 can be written as:

$u_{1}=e_{1}-y_{2}, u_{2}=e_{2}+y_{1}$
$y_{2}=S_{2} u_{2}, y_{1}=S_{1} u_{1}$

where $u_{1}, u_{2}$ are the input signals in $L_{2 e}, y_{1}, y_{2}$ are the output signals in $L_{2 e}$, and $e_{1}, e_{2}$ are the exogenous inputs in $L_{2 e}$ which are independent of the systems states. The operators $S_{1}, S_{2}$ are mappings on $L_{2 e}$ signals s.t. $S_{1}, S_{2}: L_{2 e} \longmapsto L_{2 e}$. An operator on $L_{2 e}$ is said to be $L_{2}$ stable if for an input $u$ and an output $y$ it satisfies the norm inequality: $\|y\| \leq \gamma|| u \|+b$, where $b$ and $\gamma$ are finite constants, and is said to have finite gain. For linear systems the $L_{2}$ induced gain of an operator $G$ : $L_{2 e} \mapsto L_{2 e}$ can be computed as $\gamma=\sup _{\omega>0}|G(j \omega)|$ where $G(j \omega)$ is the Fourier transform of $G$ and $\omega$ is the frequency variable. We now introduce the small gain theorem.

\section{Theorem 1- Small Gain Theorem}

The input-output interconnection in Figure 2 described by the equations (32)-(33) is bounded input bounded output (BIBO) stable if the operators $S_{1}, S_{2}$ are stable with finite gains $\gamma_{1}, \gamma_{2}$ and the product of the gains satisfy the small gain condition: $\gamma_{1} \cdot \gamma_{2}<1$. For linear operators this condition can be expressed as $\sup _{\omega \rightarrow 0}\left|S_{1}(j \omega)\right| \cdot \sup _{\omega>0}\left|S_{2}(j \omega)\right|<1$.

Next we are going to transform the bilateral teleoperation system depicted in Figure 1 and described by equations (1)-(2) into input output form as in Figure 2 and equations (32)-(33) and apply the small gain theorem to derive the stability conditions for the controllers given in (8)-(31). The system in Figure 1 can be redrawn in input-output form as in Figure 3. From the figure, the input output system transfer function $S_{1}(s), S_{2}(s)$ can be obtained as:

$$
S_{1}: \frac{\left(-C_{4}-C_{2} Z_{e}\right) e^{-s T_{2}}}{Z_{m}+C_{m}+\left(1-C_{6}\right) Z_{h}}, S_{2}: \frac{\left(C_{1}-C_{3} Z_{h}\right) e^{-s T_{1}}}{Z_{s}+C_{s}+\left(1-C_{5}\right) Z_{e}}
$$

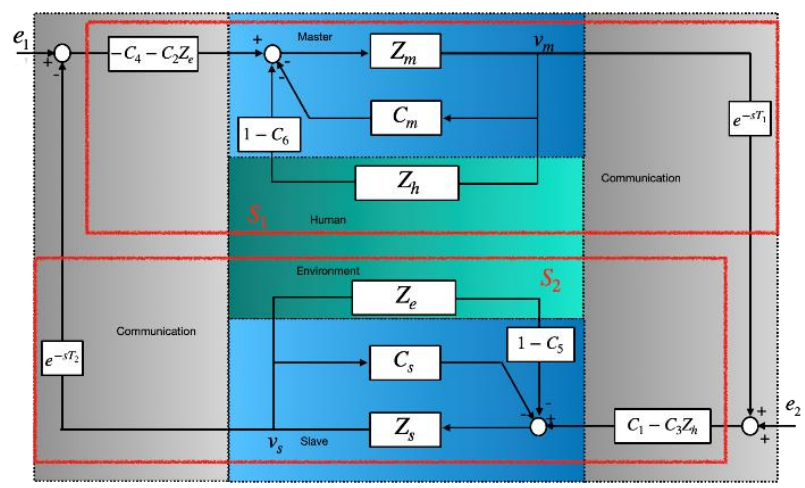

Figure 3. Input-Output Form of the Bilateral Teleoperation System 
The channel parameters in equations (8)-(31) can then be plugged in (34) to obtain the system transfer functions for different three channel architectures. The $S_{1}, S_{2}$ transfer functions for each architecture can then be written as follows.

For the P-PF architecture:

$$
S_{1}: \frac{C_{p} e^{-s T_{1}}}{Z_{m}+C_{p}}, \quad S_{2}: \frac{\left(C_{p}-C_{f} Z_{h}\right) e^{-s T_{2}}}{Z_{s}+C_{p}+b+Z_{e}}
$$

for the PF-P architecture:

$$
S_{1}: \frac{\left(C_{p}-C_{f} Z_{e}\right) e^{-s T_{1}}}{Z_{m}+C_{p}+b+C_{f} Z_{h}}, \quad S_{2}: \frac{C_{p} e^{-s T_{2}}}{Z_{s}+C_{p}}
$$

for the F-PF architecture:

$$
S_{1}: \frac{-C_{f^{\prime}} Z_{e} e^{-s T_{1}}}{Z_{m}+b+C_{f^{\prime}} Z_{h}}, S_{2}: \frac{\left(C_{p}-C_{f^{\prime}} Z_{h}\right) e^{-s T_{2}}}{Z_{s}+C_{p}+b+C_{f^{\prime}} Z_{e}}
$$

for the PF-F architecture:

$$
S_{1}: \frac{\left(C_{p}-C_{f^{\prime}} Z_{e}\right) e^{-s T_{1}}}{Z_{m}+C_{p}+b+C_{f} Z_{h}}, S_{2}: \frac{-C_{f} Z_{h} e^{-s T_{2}}}{Z_{s}+b+C_{f^{\prime}} Z_{e}}
$$

The stability conditions can obtained by determining the $L_{2}$ gains of these terms. We first solve for the controller parameters that can guarantee the stability of the P-PF architecture. From Theorem 1, the stability condition is given as: $\sup _{\omega>0}\left|S_{1}(j \omega)\right| . \sup _{\omega>0}\left|S_{2}(j \omega)\right|<1$, also both $\sup _{\omega>0}\left|S_{1}(j \omega)\right|$ and $\sup _{\omega>0}\left|S_{2}(j \omega)\right|$ should be finite. However slightly more conservative stability conditions can be obtained easily if we assume $\left|S_{1}(j \omega)\right|<1, \quad\left|S_{2}(j \omega)\right|<1, \forall \omega>1$. We have that $\sup _{\omega>0}\left|S_{i}(j \omega)\right|=\sup _{\omega>0} \sqrt{\left(S_{i}(j \omega) S_{i}(-j \omega)\right)}$. Then for the P-PF architecture we have:

$$
\left|S_{1}(j \omega)\right|=\left(\frac{k_{p}^{2}+k_{v}^{2} \omega^{2}}{M_{m}^{2} \omega^{4}-2 k_{p} M_{m} \omega^{2}+\left(k_{v}+b_{m}\right)^{2} \omega^{2}+k_{p}^{2}}\right)^{1 / 2}
$$

$$
\begin{aligned}
& \left|S_{2}(j \omega)\right|=\left(\left(C_{f}^{2} m_{h}^{2} \omega^{4}+2 C_{f} m_{h}\left(k_{p}-C_{f} k_{h}\right) \omega^{2}\right.\right. \\
& \left.\quad+\left(k_{v}-C_{f} b_{h}\right)^{2} \omega^{2}+\left(k_{p}-C_{f} k_{h}\right)^{2}\right) \\
& \quad /\left(\left(M_{s}+C_{f} m_{e}\right)^{2} \omega^{4}-2\left(C_{f} M_{e}+M_{s}\right)\left(k_{p}+C_{f} k_{e}\right) \omega^{2}\right. \\
& \left.\left.\quad+\left(k_{v}+b_{s}+C_{f} b_{e}\right)^{2} \omega^{2}+\left(k_{p}+C_{f} k_{e}\right)^{2}\right)\right)^{1 / 2}
\end{aligned}
$$

From $\left|S_{1}(j \omega)\right|<1$, the stability conditions for the master system can be written as:

$2 b_{m} k_{v}+b_{m}^{2}>2 k_{p} M_{m}$

From $\left|S_{2}(j \omega)\right|<1$, the stability conditions for the slave system can be written as:

$M_{s}+C_{f} m_{e}>C_{f} m_{h}$

$2 k_{p}>C_{f} k_{h}$

$$
\begin{aligned}
& b_{s}^{2}+2 k_{v} b_{s}+2 k_{v} C_{f} b_{h}>2\left(C_{f} M_{e}+M_{s}\right)\left(k_{p}+C_{f} k_{e}\right) \\
& +2 C_{f} m_{h}\left(k_{p}-C_{f} k_{h}\right)+C_{f}^{2} b_{h}^{2}
\end{aligned}
$$

All these conditions can be satisfied by the appropriate selection of damping injection parameters $b_{m}, b_{s}$ and the force controller gain $C_{f}$ for all possible environmental/human impedance parameters. Furthermore it can be seen that without damping injection and force controller gains it is not possible to guarantee small gain conditions and stability for all impedances. Also, due to the fact that the $L_{2}$ gain of the delay operator is unity, the delays are eliminated in the gain calculations and the stability analysis becomes delay independent. Thus the P-PF three channel controller with the controllers (23)-(25) satisfying the conditions (41)-(44) is guaranteed to be delay independent $L_{2}$ stable. Similarly, the stability condition can be derived for the PF-P architecture and will be symmetric to the P$\mathrm{PF}$ architecture. The gain functions are:

$$
\begin{aligned}
\left|S_{1}(j \omega)\right| & =\left(\left(C_{f}^{2} m_{e}^{2} \omega^{4}+2 C_{f} m_{e}\left(k_{p}-C_{f} k_{e}\right) \omega^{2}\right.\right. \\
& \left.+\left(k_{v}-C_{f} b_{e}\right)^{2} \omega^{2}+\left(k_{p}-C_{f} k_{e}\right)^{2}\right) \\
& /\left(\left(M_{m}+C_{f} m_{h}\right)^{2} \omega^{4}-2\left(C_{f} M_{h}+M_{m}\right)\left(k_{p}+C_{f} k_{h}\right) \omega^{2}\right. \\
& \left.\left.+\left(k_{v}+b_{m}+C_{f} b_{h}\right)^{2} \omega^{2}+\left(k_{p}+C_{f} k_{h}\right)^{2}\right)\right)^{1 / 2}
\end{aligned}
$$




$$
\left|S_{2}(j \omega)\right|=\left(\frac{k_{p}^{2}+k_{v}^{2} \omega^{2}}{M_{S}^{2} \omega^{4}-2 k_{p} M_{S} \omega^{2}+\left(k_{v}+b_{S}\right)^{2} \omega^{2}+k_{p}^{2}}\right)^{\frac{1}{2}}
$$

The stability conditions for the master system becomes:

$$
\begin{aligned}
& M_{m}+C_{f} m_{h}>C_{f} m_{e} \\
& 2 k_{p}>C_{f} k_{e} \\
& b_{m}^{2}+2 k_{v} b_{m}+2 k_{v} C_{f} b_{e}>2\left(C_{f} M_{h}+M_{m}\right)\left(k_{p}+C_{f} k_{h}\right) \\
& +2 C_{f} m_{e}\left(k_{p}-C_{f} k_{e}\right)+C_{f}^{2} b_{e}^{2}
\end{aligned}
$$

And for the slave it becomes:

$$
2 b_{s} k_{p}+b_{s}^{2}>2 k_{p} M_{s}
$$

On the other hand, for three channel architectures with pure force control, the stability conditions will be different. For the F-PF architecture the gain for the force controlled master system $S_{1}(j \omega)$ system with a high pass filter can be written as:

$$
\left|S_{1}(j \omega)\right|=\left(\frac{C_{f}^{2}\left(M_{e}^{2} \omega^{6}+b_{e}^{2} \omega^{4}+k_{e}^{2} \omega^{2}\right)}{M_{m}^{2} \omega^{6}+\left(b_{m}+M_{m} g\right)^{2} \omega^{4}+\left(b_{m} g\right)^{2} \omega^{2}}\right)^{\frac{1}{2}}
$$

The stability conditions can then be written as:

$$
\begin{aligned}
& M_{m}>C_{f} m_{e} \\
& b_{m} g>C_{f} k_{e} \\
& b_{m}+g M_{m}>C_{f} b_{e}
\end{aligned}
$$

Through the use of the high-pass filter and the controller gains $b_{m}, g, C_{f}$ it is possible to satisfy these conditions for all possible human impedance parameters. The slave system on the other hand which is under PF control is the same as the P-PF architecture, so the stability conditions are already provided in equations (42)-(44).

Finally, the stability conditions for the PF-F architecture is symmetric to the F-PF, and the master system gain conditions are equivalent to that of the PF$\mathrm{P}$ architecture in (47)-(49). On the other hand the slave system is force controlled, as a result the gain $\left|S_{2}(j \omega)\right|$ can be written as:

$$
\left|S_{2}(j \omega)\right|=\left(\frac{C_{f}^{2}\left(M_{h}^{2} \omega^{6}+b_{h}^{2} \omega^{4}+k_{h}^{2} \omega^{2}\right)}{M_{S}^{2} \omega^{6}+\left(b_{S}+M_{S} g\right)^{2} \omega^{4}+\left(b_{S} g\right)^{2} \omega^{2}}\right)^{\frac{1}{2}}
$$

The stability conditions can then be written as:

$$
\begin{aligned}
& M_{s}>C_{f} m_{h} \\
& b_{s} g>C_{f} k_{h} \\
& b_{s}+g M_{s}>C_{f} b_{h}
\end{aligned}
$$

Now that the conditions for delay independent stability of all three channel architectures have been demonstrated, in the next section we are going to discuss the transparency of different three channel architectures, while taking into account the controller modifications we have proposed in this section. The transparency analysis will also serve as a guide in the selection of the controller parameters for performance.

\section{TRANSPARENCY ANALYSIS}

Transparency refers to the performance of a teleoperation system. A teleoperation system should ideally become transparent to the user, meaning that it should provide a direct (kinesthetic) coupling between the master and the environment the slave robot is in contact with, without exhibiting any extra dynamics. The impedance of the environment should be transmitted to the operator one to one. Although this in theory is impossible to achieve perfectly under time delays, the degree to which it can be achieved can be measured through hybrid matrices. Kinesthetic coupling implies that at all times the position/velocities of the robots should be equal (velocity/position tracking) and the forces acting on the robots should be equal but in opposite directions (force reflection). These goals can be written in terms of the equations:

$$
\begin{aligned}
V_{m}(t) & =V_{s}(t) \\
F_{m}(t) & =-F_{s}(t)
\end{aligned}
$$

It was demonstrated in [6] that, these goals can be written in terms of a hybrid matrix which can be obtained in frequency domain by solving for the relationship between these variables from the system dynamics provided in (1)-(2). The hybrid matrix $\boldsymbol{H}$ is of the form:

$\left[\begin{array}{c}F_{m}(s) \\ V_{s}(s)\end{array}\right]=\boldsymbol{H}\left[\begin{array}{c}V_{m}(s) \\ F_{s}(s)\end{array}\right]=\left[\begin{array}{ll}h_{11} & h_{12} \\ h_{21} & h_{22}\end{array}\right]\left[\begin{array}{c}V_{m}(s) \\ F_{s}(s)\end{array}\right]$

where $h_{i j}$ are the hybrid matrix elements. The ideal kinesthetic coupling, or transparency conditions in (59)-(60) are then replicated if the hybrid matrix is written as:

$\left[\begin{array}{c}F_{m}(s) \\ V_{s}(s)\end{array}\right]=\left[\begin{array}{ll}0 & 1 \\ 1 & 0\end{array}\right]\left[\begin{array}{r}V_{m}(s) \\ -F_{s}(s)\end{array}\right]$

These ideal conditions are practically very difficult to achieve for the whole spectrum, however can be approximated by transparency optimized control architectures such as the four channel architecture when communication delays are not present. For instance practically $h_{11}$ represents the "operationality" of the teleoperation system [19], which is a measure of the impedance of the teleoperation system as perceived by the master, and in practice is almost impossible to make 0 . Therefore it is common to represent the ideal operationality by the value $\frac{C_{f}}{s}$, which is the impedance of a mass with a coefficient determined by the force controller gain. By increasing the force controller gain, it is possible to decrease operationality but this would 
be at the cost of stability. Furthermore, when delay is introduced to the system, it has been demonstrated that ideal transparency can not be achieved. The development of the three channel control architectures was a result of the need for transparency improvement under time delays. Hashtrudi-Zaad and Salcudean [9][10] demonstrated that a transparency improvement as well as guaranteed stability could be achieved with the three channel architecture. But due to the existence of time delay in the communication channels they modified the transparency conditions and introduced the concept of delayed transparency conditions:

$\left[\begin{array}{c}F_{m}(s) \\ V_{s}(s)\end{array}\right]=\left[\begin{array}{cc}0 & e^{ \pm T s} \\ e^{ \pm T s} & 0\end{array}\right]\left[\begin{array}{c}V_{m}(s) \\ -F_{s}(s)\end{array}\right]$

which also take into consideration the delay elements and meaning that the robot velocities and forces can be tracked with a certain amount of time delay. The matrix elements in (63) can be noncausal in the hybrid matrix with negative time delay for certain three channel architectures, and to solve that problem the input and output effort/flow variables can be exchanged. The resulting matrix will be the alternate or inverse hybrid matrix $\boldsymbol{G}$ [18]:

$\left[\begin{array}{r}V_{m}(s) \\ -F_{S}(s)\end{array}\right]=\boldsymbol{G}\left[\begin{array}{c}F_{m}(s) \\ V_{s}(s)\end{array}\right]=\left[\begin{array}{ll}g_{11} & g_{12} \\ g_{21} & g_{22}\end{array}\right]\left[\begin{array}{c}F_{m}(s) \\ V_{s}(s)\end{array}\right]$

the delayed transparency conditions on the inverse hybrid matrix can be obtained as:

$$
\left[\begin{array}{c}
V_{m}(s) \\
-F_{s}(s)
\end{array}\right]=\left[\begin{array}{cc}
0 & e^{\mp T s} \\
e^{\mp T s} & 0
\end{array}\right]\left[\begin{array}{c}
F_{m}(s) \\
V_{s}(s)
\end{array}\right]
$$

Thus, depending on the control architecture and time delay the appropriate matrix hybrid (63) or inverse hybrid matrix (65) is selected to analyze transparency. The magnitudes of the ideal hybrid and inverse hybrid matrices under time delay should be the same with the no time delay case as the delay operator has unity gain. Next, transparency analyses will be performed using the delayed transparency conditions for the three channel architectures under time delay with and without the proposed stability modifications and the performance of different architectures will be compared using the hybrid/inverse hybrid matrix frequency responses. Possible performance improvements and stability/transparency trade-offs will also be discussed. The hybrid matrix elements for the general Lawrence architecture as proposed in (1),(2) and Figure 1 can be obtained as:

$$
\begin{aligned}
& h_{11}=\frac{C_{1} C_{4} e^{-S T_{1}} e^{-s T_{2}}+\left(Z_{m}+C_{m}\right)\left(Z_{S}+C_{S}\right)}{\left(Z_{S}+C_{S}\right)\left(1-C_{6}\right)-C_{3} C_{4} e^{-S T_{1}} e^{-S T_{2}}} \\
& h_{12}=\frac{C_{2} e^{-S T_{2}}\left(Z_{S}+C_{S}\right)-C_{4} e^{-S T_{2}}\left(1-C_{5}\right)}{\left(Z_{S}+C_{S}\right)\left(1-C_{6}\right)-C_{3} C_{4} e^{-S T_{1}} e^{-S T_{2}}}
\end{aligned}
$$

$$
\begin{aligned}
& h_{21}=\frac{-C_{1} e^{-s T_{1}}\left(1-C_{6}\right)-C_{3} e^{-s T_{1}}\left(Z_{m}+C_{m}\right)}{\left(Z_{S}+C_{S}\right)\left(1-C_{6}\right)-C_{3} C_{4} e^{-s T_{1} e^{-s T_{2}}}} \\
& h_{22}=\frac{\left(1-C_{5}\right)\left(1-C_{6}\right)-C_{2} C_{3} e^{-s T_{1}} e^{-s T_{2}}}{\left(Z_{S}+C_{S}\right)\left(1-C_{6}\right)-C_{3} C_{4} e^{-s T_{1}} e^{-s T_{2}}}
\end{aligned}
$$

On the other hand, the inverse hybrid matrix elements can be similarly obtained as:

$$
\begin{aligned}
& g_{11}=\frac{C_{1} C_{4} e^{-s T_{1}} e^{-s T_{2}}+\left(Z_{m}+C_{m}\right)\left(Z_{S}+C_{S}\right)}{\left(Z_{m}+C_{s}\right)\left(C_{5}-1\right)-C_{1} C_{2} e^{-s T_{1}} e^{-s T_{2}}} \\
& g_{12}=\frac{C_{2} e^{-s T_{2}}\left(Z_{S}+C_{S}\right)-C_{4} e^{-s T_{2}}\left(1-C_{5}\right)}{\left(Z_{m}+C_{S}\right)\left(C_{5}-1\right)-C_{1} C_{2} e^{-s T_{1}} e^{-s T_{2}}} \\
& g_{21}=\frac{-C_{1} e^{-s T_{1}}\left(1-C_{6}\right)-C_{3} e^{-s T_{1}}\left(Z_{m}+C_{m}\right)}{\left(Z_{m}+C_{S}\right)\left(C_{5}-1\right)-C_{1} C_{2} e^{-s T_{1}} e^{-s T_{2}}} \\
& g_{22}=\frac{-\left(1-C_{5}\right)\left(1-C_{6}\right)+C_{3} e^{-s T_{1}} e^{-s T_{2}}}{\left(Z_{m}+C_{S}\right)\left(C_{5}-1\right)-C_{1} C_{2} e^{-s T_{1}} e^{-s T_{2}}}
\end{aligned}
$$

For the PF-P and F-PF architectures the hybrid matrices and for the P-PF and PF-F architectures inverse hybrid matrices will be utilized in this paper to take causality into account. Figure 4 has the magnitude frequency responses of hybrid matrices for PF-P and F-PF architectures and Figure 5 has the frequency responses for the PF-F and P-PF architectures under 1s time delays between each robot. Both the conventional and modified controllers are utilized. For the conventional controllers the parameters used are $k_{p}=900, k_{v}=$ $60, b_{m}=b_{s}=0, g=0, Z_{m}=Z_{s}=0.4 / \mathrm{s}$. and in the modified control architectures the controller parameters have been selected as in Table 1 .

For the PF-P and F-PF architectures the hybrid matrices and for the P-PF and PF-F architectures inverse hybrid matrices will be utilized in this paper to take causality into account. Figure 4 has the magnitude frequency responses of hybrid matrices for PF-P and F-PF architectures and Figure 5 has the frequency responses for the PF-F and P-PF architectures under 1s time delays between each robot. Both the conventional and modified controllers are utilized. For the conventional controllers the parameters used are $k_{p}=900, k_{v}=$ $60, b_{m}=b_{s}=0, g=0, Z_{m}=Z_{s}=0.4 / \mathrm{s}$. and in the modified control architectures the controller parameters have been selected as in Table 1 .

Table 1. Modified controller parameters in the transparency analysis

\begin{tabular}{|c|l|l|l|l|l|l|}
\hline Architecture & $k_{p}$ & $k_{v}$ & $b_{m}$ & $b_{s}$ & $C_{f}$ & $g$ \\
\hline P-PF & 900 & 60 & 10 & 150 & 0.1 & 0 \\
\hline PF-P & 900 & 60 & 150 & 10 & 0.1 & 0 \\
\hline F-PF & 900 & 60 & 150 & 150 & 0.1 & 0.1 \\
\hline PF-F & 900 & 60 & 150 & 150 & 0.1 & 0.1 \\
\hline
\end{tabular}


Without stability modifications it can be seen from Figure 4 that PF-P architecture guarantees the transparency conditions for $H_{12}, H_{22}$ for all frequencies and for $H_{21}$ within the controller bandwidth. This means that this architecture can guarantee good position and force tracking within the controller bandwidth. However $H_{11}$ response which represents operationality, shows large magnitudes and variations at low frequencies and a mass like first order system response for high frequencies. The $H_{11}$ response is ideal for high frequencies but for low frequencies the magnitude is much larger than that of a mass. This implies that during free motion at low frequencies the perceived impedance of the teleoperation system will not be like a mass and the operator will feel a large damping. Introduction of stability modifications in the form of extra damping injection and lowered force control gain mainly affects the $H_{11}$ term, and increases the magnitude at low frequencies and the operationality even more. The conventional F-PF architecture on the other hand, has ideal responses in $H_{11}, H_{12}, H_{21}$ and an almost ideal response in $H_{22}$. While the magnitude in $H_{22}$ is not zero it can be considered ideal as the magnitude is negligible. $H_{11}$ on the other hand, is an ideal mass-like first order response. When the modifications in the form of damping injection and force filters are applied, again the only deterioration is seen in the $H_{11}$. The low frequency magnitude is increased more uniformly but the resulting magnitude is still much smaller than the PF-P architecture at low frequencies, meaning that the operationality of the F-PF architecture would be much less and the system would feel lighter and faster to the operator. Increasing the damping injection terms and high pass filter cutoff frequency improves the $\mathrm{H}_{22}$ response leading to better position tracking. However, since the PF-P architecture has an ideal $\mathrm{H}_{22}$ response this would imply that the PF-P architecture would have an improved position tracking over the F-PF architecture.

Figure 5 shows the inverse hybrid matrix responses for P-PF and PF-F architectures. Since there is a symmetry between hybrid and inverse hybrid matrices, when the corresponding $G_{11}-H_{22}, \quad G_{12}-H_{21}, \quad G_{21}-H_{12}$, $G_{22}-H_{11}$ magnitude responses are compared, P-PF and PF-P architectures are very similar and F-PF and PF-F architectures are very similar in terms of performance. As the port variables in equations (61),(64) are inverted, the phase relationship between the responses will also be inverted, meaning that if the master position is leading and master force is lagging in F-PF architecture, for the PF-F architectures, the master position would be lagging and master force would be leading.
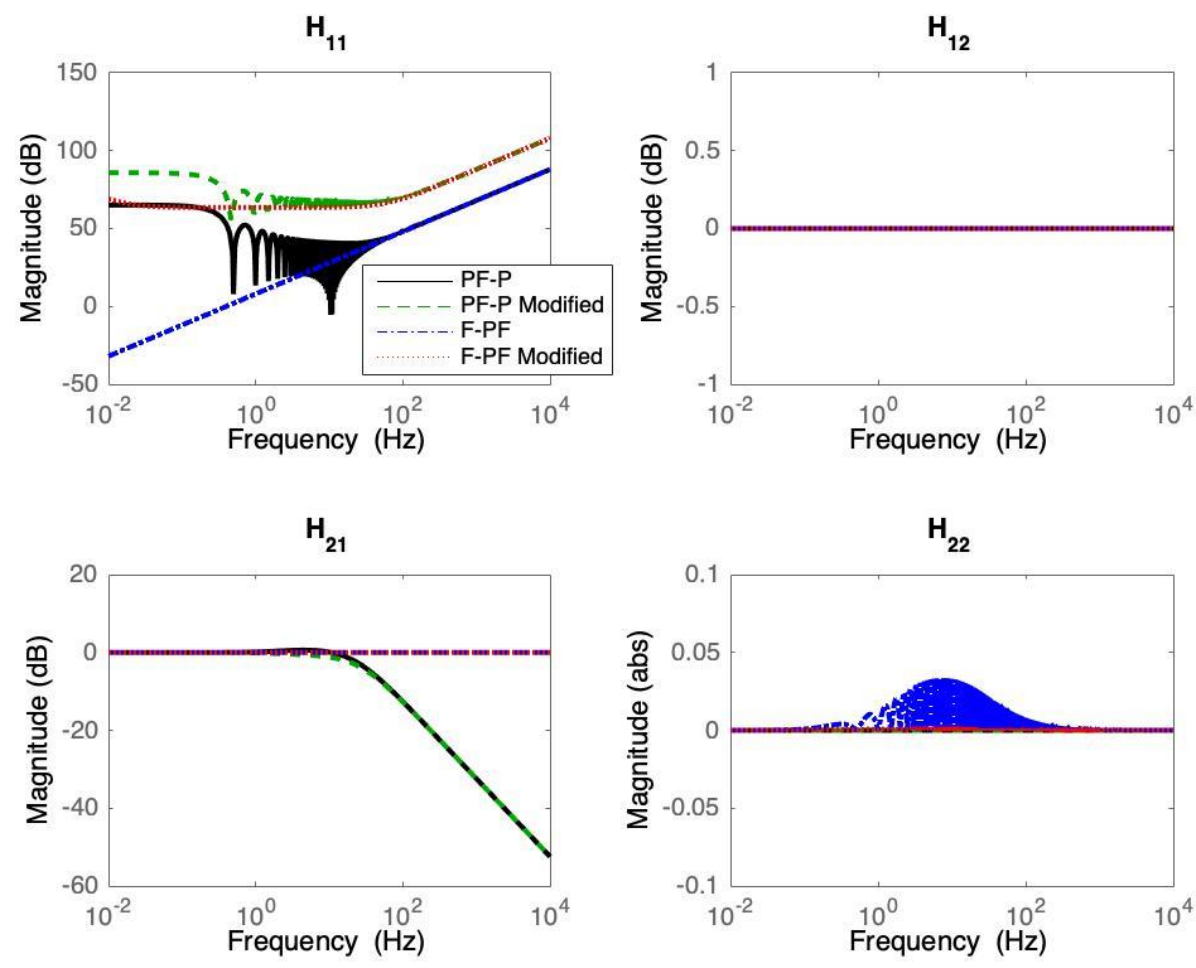

Figure 4. Frequency Responses of the Hybrid Matrix Elements for Three Channel PF-P and F-PF Architectures 

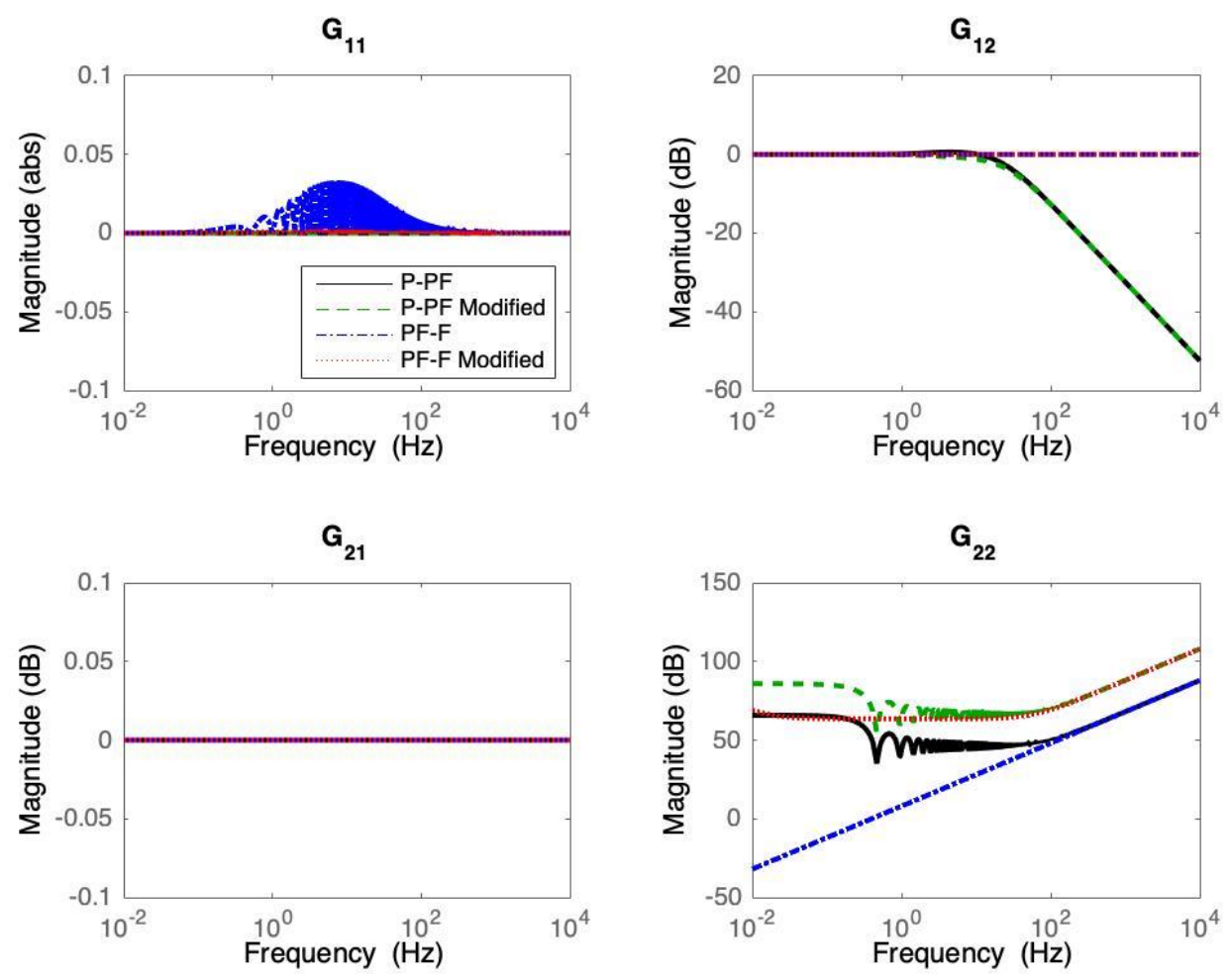

Figure 5. Frequency Responses of the Inverse Hybrid Matrix Elements for Three Channel P-PF and PF-F Architectures

Figures 4-5 together show that there is a trade-off between stability and the operationality of the teleoperation system. Increasing the damping injection terms, high pass filter cut-off frequency, and decreasing force controller gain for robustness increases the operationality of the system, meaning that it deteriorates performance. Since the transparency of the architectures with same parameters are similar, the environment and operator impedance assumptions in a given teleoperation setup would actually be critical in the selection of the control architecture. Previous section introduced the stability criteria for different architectures, and these criteria require different control parameter selections for the same environment/operator impedance pairs. As a result, these different controller parameters would result in different transparency characteristics under the same experiment settings. Certain impedance-environment pairs would favor certain architectures, for instance if the environment has a stiffness less than the operator, F-PF and PF-P architectures would be more favorable as the required damping terms would be less, resulting in better transparency. However, if the environment is stiffer than the operator, than the PF-F and P-PF architectures would be more favorable. Experiments have been conducted to verify the validity of both the stability and transparency analyses provided so far and the results will be presented in the next section.

\section{EXPERIMENT RESULTS}

Experiments were conducted on a bilateral teleoperation setup consisting of two linear motors connected through a computer. The motors used in the experiments are Dunkermotoren STA1116 with high precision encoders for position measurement. An NI PCI-6321 DAQ card was used to interface the motors with the computer and the controller runs on Matlab Simulink with a sampling time of $1 \mathrm{~ms}$. Communication among the motors take place in the same control program and time delays were also created in the controller environment. In the experiments an operator holds one of the motor shafts and moves the shaft back and forth. The slave motion is constrained by a wooden block at a certain location and when the slave contacts this wooden block it is desirable that the master also comes to a stop and transmits the environmental force back to the master. The goal is for both of the robots to follow the same trajectory at all times and have the same measured force in the opposite directions during contact. The force measurements from the motors are obtained through reaction force observers as described in [20]. The experiment setup can be seen in Figure 6. 


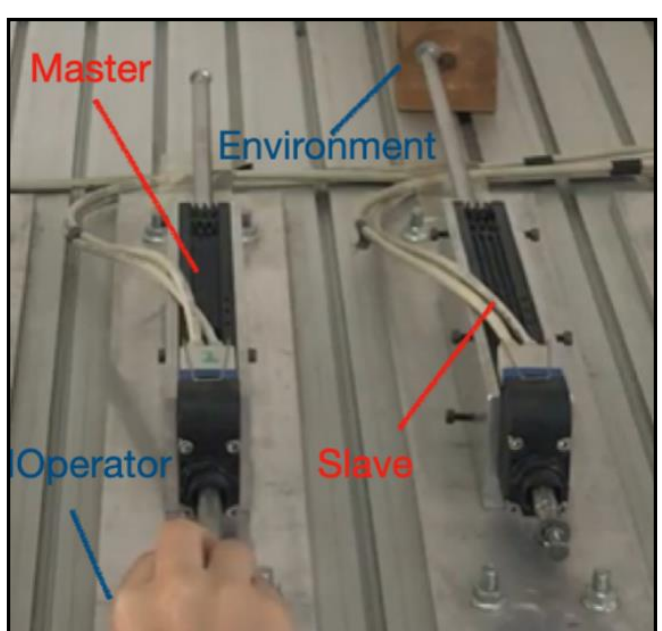

Figure 6. Bilateral Teleoperation Experiment Setup

In the experiment, both free motion and contact scenarios were tested and as a result this provides two different environments in the same experiment. During free motion, environment stiffness is zero and during contact with wood the environment stiffness can be very large: $\mathrm{K}>70000 \mathrm{~N} / \mathrm{m}$ [21]. By performing system identification using Matlab System Identification Toolbox the maximum human impedance parameters were obtained as $m_{h}=2, b_{h}=25, k_{h}=90$. The large difference in the magnitudes of the stiffness values between the human and environment creates an asymmetry between the different three channel control architectures due to the constraints on the minimum values controller parameters can take. The existence of a high a stiffness environment means that for PF-P architecture the minimum proportional control term should be $k_{p}>3500$, which is a very high controller gain resulting in high operationality, amplification of measurement noise and actuator saturation. Similarly for the F-PF architecture, the stability condition of $b_{m} g>10000 C_{f}$ would mean a very large master damping or low force controller gain and high pass cut off frequency, again resulting in a very high operationality and actuator saturation. For the PF-F architecture the same condition $k_{p}>3500$ is valid for the master, but since the L2 gain of the slave system can easily be made much smaller, according to small gain theorem, the closed loop system can be made stable for smaller $k_{p}$ values. For P-PF architecture such constraints are much less conservative with $k_{p}>45$, and as a result this is the most robust control architecture. Since the gains for all controllers could not be selected as high as the stability conditions suggest in the current experiment setup due to actuator saturation and noise amplification, the same set of controller parameters were used to compare the performances of the controllers under the same circumstances. The controller parameters were selected as in transparency section, Table 1 . Time delays between the systems were selected significantly large: $0.2 \mathrm{~s}$ single-way and $0.4 \mathrm{~s}$ round-trip. Figures 7-10 show the experiment results for each architecture.
Figure 7 shows the position and force measurements from each system when the F-PF architecture is used. In the experiment, the operator starts moving the master backwards and then forwards and at about $7 \mathrm{~s}$ contacts the environment, keeping contact until $15 \mathrm{~s}$, and then retreats the master robot and then again contacts the environment between $18 \mathrm{~s}-27 \mathrm{~s}$. Blue lines show master position and force, red lines show slave position and force, and the yellow lines show the tracking errors. The delay shifted position and force signals are used in the error calculations and are also in the plots. While the free motion performance of the control system is very good, with perfect position tracking and low operational forces, during contact it is difficult to keep the slave in contact with the environment by applying a constant force due to the violation of stability condition (53). The operator has to increase their arm stiffness to keep the robot in stable contact as environment pushes the master back. During first contact stable contact can be achieved, and good force reflection is realized, however in the second contact due to large force reflection undesirable loss of contact occurs and both position and force tracking performance deteriorates. Also the master position leads the slave position and the slave force leads the master force in this architecture.

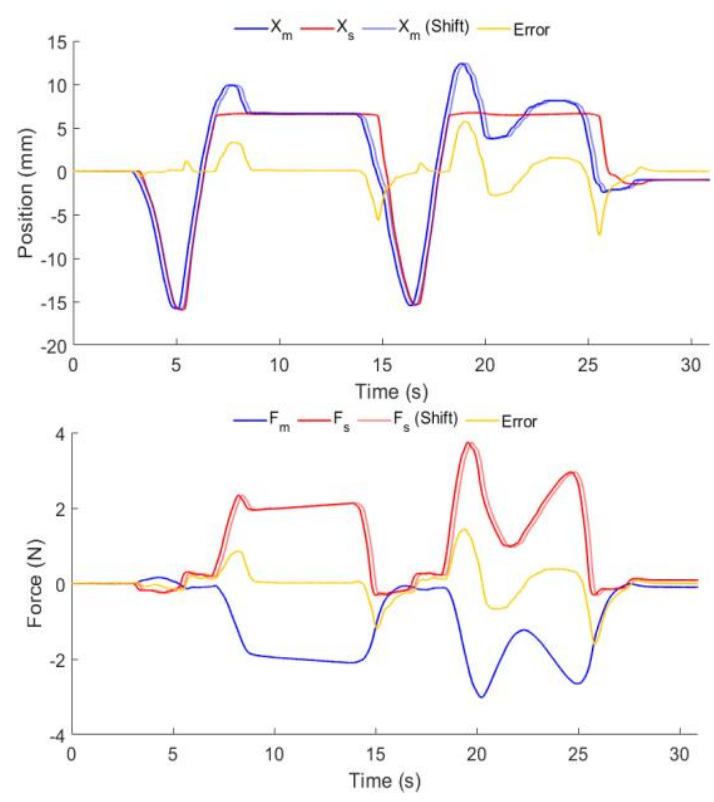

Figure 7. Position and Force responses of F-PF Architecture with 0.2s Time Delays

Figure 8 shows the experiment results when the same procedure is repeated with the PF-P architecture. Again during free motion the position tracking is good but the operational forces are higher as can be seen from the force response. This makes the system feel heavier to the operator and the same operation takes longer to complete. However just as in the previous case it is difficult to keep the robot in contact with a stiff environment and oscillations take place due to the violation of the stability condition (48), also resulting in large force errors. The shift between the robot responses is also comparatively less with this architecture. 

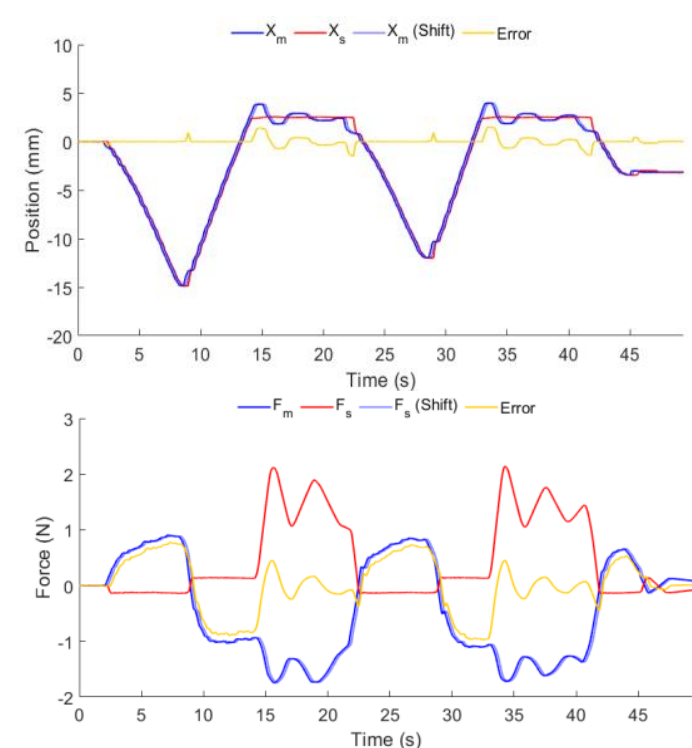

Figure 8. Position and Force responses of PF-P Architecture with 0.2s Time Delays

Figure 9 shows the experiment results when the same procedure is repeated with the PF-F architecture. With this architecture, stability can be more easily guaranteed both in free motion and contact. Just like the F-PF architecture, the free motion position tracking is very good with a delay shift between the robots, but this time the slave position leads the master position. During contact the system is more easily stabilized than PF-P architecture but some overshoot occurs during contact due to the master feeling the environment feedback with delay. However after the transient period is over perfect force reflection is achieved during contact. The force of the master leads the slave force in this architecture.

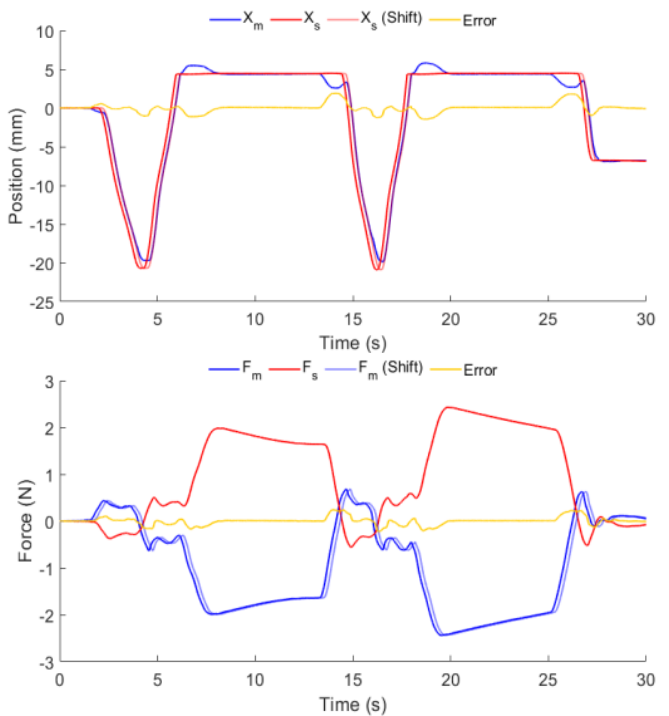

Figure 9. Position and Force responses of PF-F Architecture with $0.2 \mathrm{~s}$ Time Delays

Figure 10 shows the experiment results when the same procedure is repeated with the P-PF architecture. This architecture is the most robust and there is no overshoot in position and force tracking. The tracking results are close to perfect. However this architecture also has larger operational forces during free motion and this makes the system heavier and slower than the other architectures. Delay shift between the robots is also limited in this architecture.

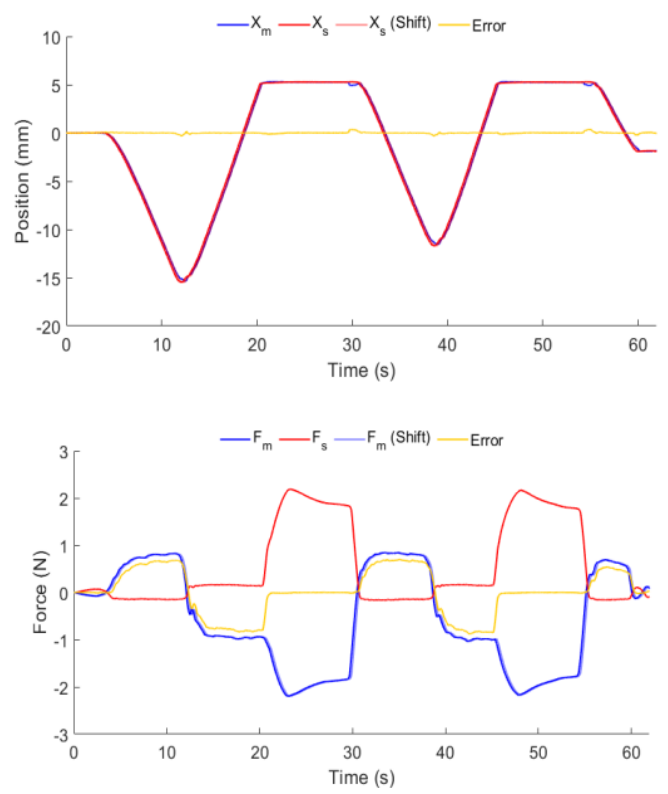

Figure 10. Position and Force responses of P-PF Architecture with $0.2 \mathrm{~s}$ Time Delays

The experiment results confirm the stability and transparency analyses provided in the previous sections. It is demonstrated that in the presence of stiff environments P-PF and PF-F architectures have advantages over PF-P and F-PF architectures in terms of stability and transparency. P-PF architecture is the most robust architecture and has the best position and force tracking performance closely followed by the PF-F architecture, however the PF-F architecture has much smaller operationality and this results in a faster and lighter teleoperation system. This is achieved by asynchronous tracking among the robots as there are shifts in the position and force responses. If a fast teleoperation system is desirable the PF-F architecture would be preferable, but if precision and synchronicity is required the P-PF architecture would be the better option. In the case of soft environment similar performances can be expected from F-PF and PF-P architectures but the delay shifts would be inverted. These architectures could be preferred if the application requires the master position to lead the slave position and if the master impedance is much greater than the environment, these architectures would be less conservative in terms of stability guarantees and therefore could be better in terms of operationality.

\section{CONCLUSIONS}

This paper has presented comprehensive transparency and stability analyses for three channel architectures that are commonly used in bilateral teleoperation applications. Controller modifications were proposed for guaranteeing delay independent stability for each architecture for the first time, and the effect of these 
modifications on transparency was also discussed. The analyses were verified with experiment results. Each architecture have their own merits and could be utilized for different applications. But it can be suggested that the PF-F and P-PF architectures are more robust with a wide range of environments. PF-F architecture can be selected for fast and light systems and P-PF architecture can be selected for operations requiring greater precision and care. Future works involve extending these analyses to other Lawrence architectures, and using robust control techniques to account for model uncertainties and nonlinearities.

\section{REFERENCES}

[1] Anderson, R. J., \& Spong, M. W. (1988, August). Bilateral control of teleoperators with time delay. In Proceedings of the 1988 IEEE International Conference on Systems, Man, and Cybernetics (Vol. 1, pp. 131-138). IEEE.

[2] Niemeyer, G., \& Slotine, J. J. (1997, April). Using wave variables for system analysis and robot control. In Proceedings of International Conference on Robotics and Automation (Vol. 2, pp. 1619-1625). IEEE.

[3] Ryu, J. H., Kwon, D. S., \& Hannaford, B. (2004). Stable teleoperation with time-domain passivity control. IEEE Transactions on robotics and automation, 20(2), 365-373.

[4] Nuño, E., Basañez, L., \& Ortega, R. (2011). Passivity-based control for bilateral teleoperation: A tutorial. Automatica, 47(3), 485-495.

[5] Lee, D., \& Spong, M. W. (2006). Passive bilateral teleoperation with constant time delay. IEEE transactions on robotics, 22(2), 269-281.

[6 Hannaford, B. (1989). A design framework for teleoperators with kinesthetic feedback. IEEE transactions on Robotics and Automation, 5(4), 426-434.

[7] Lawrence, D. A. (1993). Stability and transparency in bilateral teleoperation. IEEE transactions on robotics and automation, 9(5), 624-637.

[8] Yokokohji, Y., \& Yoshikawa, T. (1994). Bilateral control of master-slave manipulators for ideal kinesthetic coupling-formulation and experiment. IEEE transactions on robotics and automation, 10(5), 605-620.

[9] Hastrudi-Zaad, K., \& Salcudean, S. E. (1999, May). On the use of local force feedback for transparent teleoperation. In Proceedings 1999 IEEE International Conference on Robotics and Automation (Cat. No. 99CH36288C) (Vol. 3, pp. 1863-1869). IEEE.

[10] Hashtrudi-Zaad, K., \& Salcudean, S. E. (2002). Transparency in time-delayed systems and the effect of local force feedback for transparent teleoperation. IEEE Transactions on Robotics and Automation, 18(1), 108-114.
[11] Kubo, R., Iiyama, N., Natori, K., Ohnishi, K., \& Furukawa, H. (2007). Performance analysis of a three-channel control architecture for bilateral teleoperation with time delay. IEEJ Transactions on Industry Applications, 127(12), 1224-1230.

[12] Tumerdem, U. (2019). Three-channel control architecture for multilateral teleoperation under time delay. Turkish Journal of Electrical Engineering \& Computer Sciences, 27(1), 120138.

[13] Albakri, A., Liu, C., \& Poignet, P. (2013, November). Stability and performance analysis of three-channel teleoperation control architectures for medical applications.In IEEE/RSJ International Conference on Intelligent Robots and Systems (pp. 456-462). IEEE.

[14] Tumerdem, U., \& Ohnishi, K. (2009, April). Robust four channel teleoperation under time delay by damping injection. In 2009 IEEE International Conference on Mechatronics (pp. 1-6). IEEE.

[15] Tumerdem, U., \& Ohnishi, K. (2010). DelayIndependent L2 Stability of Four-Channel Bilateral Teleoperators with Damping Injection. IEEJ Transactions on Industry Applications, 130(8), 953-964.

[16] Naerum, E., \& Hannaford, B. (2009, May). Global transparency analysis of the lawrence teleoperator architecture. In 2009 IEEE International Conference on Robotics and Automation (pp. 4344-4349). IEEE.

[17] Van der Schaft, A. J., \& Van Der Schaft, A. J. (2000). L2-gain and passivity techniques in nonlinear control (Vol. 2). London: Springer.

[18] Hashtrudi-Zaad, K., \& Salcudean, S. E. (2000, April). Analysis and evaluation of stability and performance robustness for teleoperation control architectures. In Proceedings 2000 ICRA. Millennium Conference. IEEE International Conference on Robotics and Automation. Symposia Proceedings (Cat. No. OOCH37065) (Vol. 4, pp. 3107-3113). IEEE.

[19] Iida, W., \& Ohnishi, K. (2004, March). Reproducibility and operationality in bilateral teleoperation. In The 8th IEEE International Workshop on Advanced Motion Control, 2004. AMC'04. (pp. 217-222). IEEE.

[20] Murakami, T., Yu, F., \& Ohnishi, K. (1993). Torque sensorless control in multidegree-offreedom manipulator. IEEE Transactions on Industrial Electronics, 40(2), 259-265..

[21] Kuchenbecker, K. J., Fiene, J., \& Niemeyer, G. (2006). Improving contact realism through event-based haptic feedback. IEEE transactions on visualization and computer graphics, 12(2), 219-230. 